were adapted specific to location (health centre/hospital). Surveys were undertaken across 45 health centres and 1 hospital. Although knowledge rates were acceptable, attitudes continuously fell into indifferent or negative ranges in certain domains.

A multiple tiered neonatal training programme was developed, depending on staff type (midwife, nurse or doctor) comprising of a classroom-based residential component, shadowing at a tertiary neonatal facility and an extensive mentorship period. Teaching materials, assessments and logbooks were prepared, tailored to conditions commonly encountered and resources available locally, written in English and translated into Khmer. Where resources were deficient, plans to supply equipment were initiated. Development of a neonatal care unit was commenced.

Conclusions An assessment tool called a 'KAPES survey' was created to comprehensively assess neonatal services in rural Cambodia. A comprehensive multiple-tier neonatal training programme was developed and adapted to specific needs. Initial data guided implementation of the strategy to decrease the NMR. Repeating the survey will evaluate progress of the intervention. Ultimately, this tool and programme could be replicated across the country and may be useful in other middle to low-income countries.

\section{P439 A RETROSPECTIVE AUDIT LOOKING AT ECHO FINDINGS IN BABIES BORN WITH DOWN SYNDROME IN A TERTIARY NEONATAL UNIT}

Sarah Kyne*, Anna Curley. Neonatal Department, National Maternity Hospital, Holles Street, Dublin, Ireland

\subsection{6/archdischild-2019-epa.775}

Aims Down Syndrome (T21) is the most frequent chromosomal anomaly, occurring in one per 546 live births in Ireland ${ }^{1}$. Population cohort studies show cardiac anomalies are found in $57 \%$ of babies diagnosed with T21 with atrio-ventricular septal defects being the most common presentation ${ }^{2}$. Routine antenatal scans have meant that some babies with T21 are diagnosed with cardiac anomalies before birth. Our local guidelines emphasise that all children diagnosed with T21 have an early postnatal echocardiogram and referral to consultant cardiologist. Our aim was to assess whether we were meeting this objective and to describe the range of cardiac diagnoses in our cohort.

Methods Setting was a tertiary maternity hospital (8,000 birth per annum). This was a retrospective chart audit of all babies born 2016-2017 with a diagnosis of T21.

\begin{tabular}{ll} 
Abstract P439 Table 1 & \\
\hline Cardiac Anomaly & Number of Babies \\
\hline AVSD & 9 \\
ASD & 7 \\
Pulmonary hypertension & 7 \\
VSD & 6 \\
PDA & 6 \\
Normal Echo & 5 \\
\hline
\end{tabular}

Results 37/42 babies diagnosed with T21 were included in the study. 54\% of these babies needed admission to the baby unit. Mean age at birth was $35+6$ weeks, birth weight $2.9 \mathrm{~kg} .12$ babies were antenatally diagnosed. Cardiac findings at the 20week scan led to $67 \%$ of antenatal diagnoses. Of the four babies antenatally diagnosed with no cardiac findings at 20week scan; 1 had a small ASD on postnatal echo and the second had no cardiac findings. All babies, regardless of cardiac findings on clinical examination, received a postnatal echo. 36 received an echo while still an inpatient. $87 \%$ had at least one cardiac anomaly and $24 \%$ of the babies had an AVSD confirmed on echo. The table 1 below shows the top 7 (of 20) cardiac diagnoses from postnatal echo.

Conclusion One third of babies were diagnosed antenatally despite a high incidence of postnatal cardiac findings Postnatally $87 \%$ of babies had a cardiac anomaly with AVSD in $24 \%$. There is a wide variety of cardiac findings in this cohort of babies.

\section{REFERENCES}

1. Johnson Z, Lillis D, Delany V, et al. (1996) The epidemiology of Down syndrome in four counties in Ireland 1981-90. J Public Health Med 18:78-86

2. Weijerman ME, van Furth AM, Vonk Noordegraaf A, van Wouwe JP, Broers CJ, Gemke RJ. Prevalence, neonatal characteristics, and first-year mortality of Down syndrome: a national study. J Pediatr.2008;152(1):15-19

\section{P440 CADIAC NEONATAL LUPUS}

${ }^{1}$ Afef Ben Thabet, ${ }^{1}$ Mouna Loukil, ${ }^{1}$ Amel Ben Hamed, ${ }^{1}$ Chiraz Regaieg*, ${ }^{1}$ Manel Charfi, ${ }^{2}$ Mohamed Derbel, ${ }^{1}$ Nedia Hmida, ${ }^{1}$ Ridha Regaieg, ${ }^{3}$ Dorra Abid, ${ }^{2}$ Kais Chaabene, ${ }^{3}$ Samir Kamoun, ${ }^{1}$ Amira Bouraoui, ${ }^{1}$ Abdellatif Gargouri. ${ }^{1}$ Department of neonatology, Hedi Chaker Hospital, Sfax, Tunisia; ${ }^{2}$ Departement of Obstetrics and Gynecology, Hedi Chaker Hospital, Sfax, Tunisia; ${ }^{3}$ Cardiology Department , HediChaker Hospital, Sfax, Tunisia

\subsection{6/archdischild-2019-epa.776}

Background Neonatal lupus erythematosus (NLE) is an uncommon acquired autoimmune disease caused by transplacental passage of maternal anti-SSA/Ro and anti-SSB/La antibodies. The most common effects of NLE include cutaneous involvement and congenital heart block (CHB). The death rate of complete congenital heart block (CCHB), which is the most severe clinical manifestation, is as high as $20 \%$ to $30 \%$. That's why, early recognition of infants at risk is important.

Objectives To investigate the clinical features and long-term prognosis of cardiac NLE.

Methods A retrospective descriptive study of nine cases with cardiac events of neonatal lupus over a 15 -year period from January 2003 to December 2018. The diagnostic criteria required the presence of clinical symptoms and positive autoantibodies both in the mother and her child.

Results There were 9 patients (male: female ratio of 6:3). Sjögren syndrome was the most common maternal involvement. CCHB was detected in 7 patients of whom 2 patients had an antenatal diagnosis. Two other patients were presented with a cardiomyopathy with immediate heart failure in one case. Antenatal treatment with glucocorticoids and/or hydroxychloroquine was used in 3 cases (maternal connective disease in 2 cases and CCHB in 1 case). After birth, 2 patients were treated with isoprenaline and 6 of the 8 patients diagnosed with the $\mathrm{CHB}$ received single-chamber pacemaker implants. Immediate evolution was favorable for all patients. During the follow-up, two infants died. A cardiomyopathy appeared in 
one patient with $\mathrm{CCHB}$ one year after pace maker implanting.

Conclusions CCHB is the most serious manifestation of the NLE associated with significant morbidity and mortality. Early diagnosis can lead to early treatment avoiding in some cases the onset of CCHB.

\section{P441 YIELD OF URINE FOR CMV SCREENING TEST FOR ASYMPTOMATIC MICRO-CEPHALIC INFANTS1}

Haroon Usman*, Prof Martin White. Neonatology Department, Coombe Women and Infants University Hospital, Dublin, Ireland

\subsection{6/archdischild-2019-epa.777}

Background Congenital CMV infection is the leading cause of nonhereditary SNHL and other long-term neurodevelopmental disabilities. Its prevalence is $0.6 \%$ in developed countries. Approximately $10 \%$ infants have symptoms at birth. Among apparently asymptomatic infants, approximately 10 to $15 \%$ experience SNHL

Objective To determine the utility and cost-effectiveness of urine CMV PCR testing among infants with OFC $<9^{\text {th }}$ centile. To evaluate the frequency of alternative aetiologies of microcephaly in tested infants

Methods A retrospective review was conducted on all infants with a diagnosis of microcephaly for whom urine for CMV or TORCH screen was sent during July 2017-July 2018 in Coombe Women and Infants University Hospital ${ }^{1}$, Dublin, Ireland. Data was collected for various characteristics such as gestational age, ethnicity, parity, mother's serology, mother's lifestyle, reason of screening, anthropometric measurement/ Centile and cranial ultra sound result.

Results Between July 2017-July 2018, out of 8200 infants born in CWIUH, $72(0.87 \%)$ infants were screened. Of these, 56 infants had urine for CMV performed, and 16 infants had both TORCH titer and Urine for CMV testing performed. There were only 3 positive CMV cases (4.16\%). Male infants were $32(44.44 \%)$ and female infants were 40 (55.55\%). 25 infants were first born baby in their family. Mothers of 33 infants were drinking, smoking or using drugs during pregnancy. 29 infants underwent cranial ultrasound, of which 9 had abnormal results.

Conclusions By plotting on actual GA rather than on term line for term babies, we could avoid screening of $12.5 \%$ babies. Ethnicity factor is irrelevant. Considering the low incidence of congenital CMV infection (0.8\%), current local guidelines for congenital CMV infection screening is cost effective (yield $4.16 \%$ ). This study serves as a reminder to periodically examine testing practices and patient population to maximize cost-effectiveness.

\section{P442 ECHOCARDIOGRAPHY IN THE TERTIARY SURGICAL NEONATAL INTENSIVE CARE UNIT - A SERVICE EVALUATION PROJECT}

Katherine Millard, Emma Spencer*. Queen's Medical Centre, Nottingham, UK

\subsection{6/archdischild-2019-epa.778}

Introduction The use of echocardiography in neonatal care is currently the subject of significant and growing interest. It is well-recognised that there is wide variation in the approach to neonatal circulatory management, and that many of the commonly-assessed clinical parameters are of limited value in assessing adequacy of circulation. Much of the recent research interest has focussed on the potential uses of echocardiography in haemodynamic assessment of the preterm infant. However, the diverse group of newborn infants with surgical problems are also prone to cardiovascular compromise, particularly in the perioperative period. Additionally they may have structural cardiac defects, commonly as part of a syndrome or association. The echocardiographic needs of this cohort may therefore be different from those of the typical preterm population.

Aim The purpose of this study was to evaluate the use of echocardiography in a tertiary surgical neonatal intensive care unit to better understand current practice and the needs of the population, and to inform future service planning.

Methods We retrospectively reviewed all echocardiograms performed on our 17-cot unit over a 12-month period from November 2018 to October 2018. Demographic and clinical data were collected for all patients.

Results Over the study period 182 echocardiograms were performed on 122 infants. Echocardiograms were performed primarily by technicians, with $82 \%$ being performed within one day of the request being submitted. $43 \%$ of studies were carried out in term infants, with $52 \%$ taking place within the first three days of life. The most common indications were evaluation of a murmur in a preterm infant (22\%), known congenital or genetic anomalies (20\%) and functional cardiac assessment (13\%). A PDA was identified in 59\% of initial scans. Structural anomalies identified included ASD (27\%), VSD (17\%), AVSD (0.8\%), Tetralogy of Fallot (2.5\%) and coarctation of the aorta $(0.8 \%)$. Abnormal findings related to function or haemodynamics were reported in $19 \%$.

Conclusions Echocardiography is a commonly-performed investigation in a tertiary surgical neonatal intensive care unit. Both structural and functional assessments are important in the care of infants with surgical problems. This study provides baseline data on current practice and suggests a need for surgical neonatal services to consider adequate provision for echocardiography in any future service planning.

\section{P443 HEREDITARY THROMBOPHILIA IN NEWBORN}

Afef Ben Thabet, Rim Zaaghdoud, Manel Charfi, Chiraz Regaieg*, Amel Ben Hmed, Nedia Hmida, Amira Bouraoui, Abdellatif Gargouri. Department of neonatology, Hedi Chaker Hospital, Sfax, Tunisia

\subsection{6/archdischild-2019-epa.779}

Background Hereditary Thrombophilia refers to the propensity to develop thrombosis. A homozygous abnormality or combination of two heterozygous abnormal factors can lead to clinically apparent thrombotic disorders at an early age. The aim of our study was to analyse the epidemiology, clinical presentation, causes, and management of hereditary thrombophilia in newborn.

Methods It's a retrospective study of all cases of hereditary thrombophilia registered in the neonatal intensive care unit of Sfax between 2014 and 2017.

Results Seven full term newborns were included. They were 5 female and 2 male. Common clinical features were seizures, acute ischemic limb, mesenteric ischemia and skin necrosis 